\title{
Improved Dried Blood Spot-Based Metabolomics Analysis by a Postcolumn Infused-Internal Standard Assisted Liquid Chromatography-Electrospray Ionization Mass Spectrometry Method
}

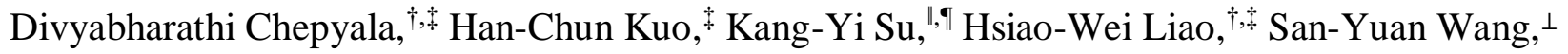
Surendhar Reddy Chepyala, ${ }^{\#}$ Lin-Chau Chang, ${ }^{*},{ }^{\dagger}$ and Ching-Hua Kuo ${ }^{*}, \dagger,+, \S$

$\dagger$ School of Pharmacy, College of Medicine, National Taiwan University, Taipei 10050, Taiwan

$\$$ The Metabolomics Core Laboratory, Centers of Genomic and Precision Medicine, National Taiwan University, Taipei 10055, Taiwan

"Department of Clinical Laboratory Sciences and Medical Biotechnology, College of Medicine, National Taiwan University, Taipei 10617, Taiwan

${ }^{\text {II }}$ Department of Laboratory Medicine, National Taiwan University Hospital, Taipei 10002, Taiwan

$\S^{\S}$ Department of Pharmacy, National Taiwan University Hospital, Taipei 10051, Taiwan

${ }^{\perp}$ Master Program in Clinical Pharmacogenomics and Pharmacoproteomics, College of Pharmacy, Taipei Medical University, Taipei 11031, Taiwan

\# Institute of Chemistry, Academia Sinica, Taipei 115, Taiwan

\section{*Corresponding authors:}

1. Prof. Ching-Hua Kuo

School of Pharmacy, College of Medicine, National Taiwan University, No. 33, Linsen S. Rd., Zhongzheng Dist., Taipei City 10050, Taiwan

Tel.: +886-2-33668766; Fax: +886-2-23919098

E-mail: kuoch@ntu.edu.tw

2. Dr. Lin-Chau Chang

School of Pharmacy, College of Medicine, National Taiwan University, No. 33, Linsen S. Rd., Zhongzheng Dist., Taipei City 10050, Taiwan

Tel.: +886-2-33668197; Fax: +886-2-23919098

E-mail: linchauchang@ntu.edu.tw 
This SI contains the following:

Section S-1. DBS sample preparation procedure using Hybrid SPE technique.

Figure S-1. The overlaid total ion chromatograms of DBS (green color) and plasma (red color) samples obtained from precursor ion scan $\mathrm{m} / \mathrm{z} 184$.

Figure S-2. (A) The representative LC-MS chromatogram of target metabolites and (B) the PCIIS chromatogram for the PCI-IS method.

Figure S-3. Correlations between signal intensity and DBS spot volume before and after PCI-IS correction.

Table S-1. Metabolites with significantly different levels ( $p$-values $<0.05$ ) in DBS samples between APAP-induced liver toxicity and control groups.

Section S-1. DBS Sample Preparation Procedure using Hybrid SPE Technique. The blood samples collected in EDTA tubes were used to spot on DBS cards within 15 min of blood collection. Twenty microliters of the blood sample was spotted onto a DBS card, and the spotted cards were air-dried for $2 \mathrm{~h}$. After drying, the whole blood spot was cut into a clean Eppendorf tube using a 6-mm manual puncher by punching several times. Then, $300 \mu \mathrm{L}$ of $1 \%$ citric acid in $80 \%$ ACN was added to extract the cut blood spot using ultrasonication for $30 \mathrm{~min}$. After sonication, the samples were centrifuged for $5 \mathrm{~min}$ at $15000 \mathrm{rcf}$ and subjected to Hybrid SPE.

Hybrid SPE sample preparation was performed on a Waters vacuum manifold (MA, USA). The Hybrid SPE (1 mL, $100 \mathrm{mg}$, Sigma, St. Louis, MO, USA) cartridges were loaded with the sample solution containing $270 \mu \mathrm{L}$ of $1 \%$ citric acid in $80 \%$ ACN after centrifugation. Then the sample solution was eluted slowly by applying the pressure. The eluted samples were evaporated under nitrogen gas and dried samples were reconstituted with $150 \mu \mathrm{L}$ of $50 \% \mathrm{MeOH}$. The reconstituted samples were vortexed and then filtered through a $0.2 \mu \mathrm{m}$ regenerated cellulose membrane (Sartorius, Göttingen, Germany) and analyzed by UHPLC-ESI-MS/MS system.

Twenty metabolites selected based on a wide coverage of $\log \mathrm{P}$ values and metabolite categories were used to evaluate the performance of hybrid SPE in removing lipids for DBS-based metabolomics studies. The metabolite extract obtained from $80 \%$ ACN was further treated by hybrid SPE. Although the hybrid SPE technique was able to capture the phospholipids via a Lewis acid-base interaction with zirconia sites, the recovery of target analytes was greatly decreased. The adjustments of sample solution and elution buffer and the addition of a second elution step failed to significantly improve the recovery of target metabolites. 


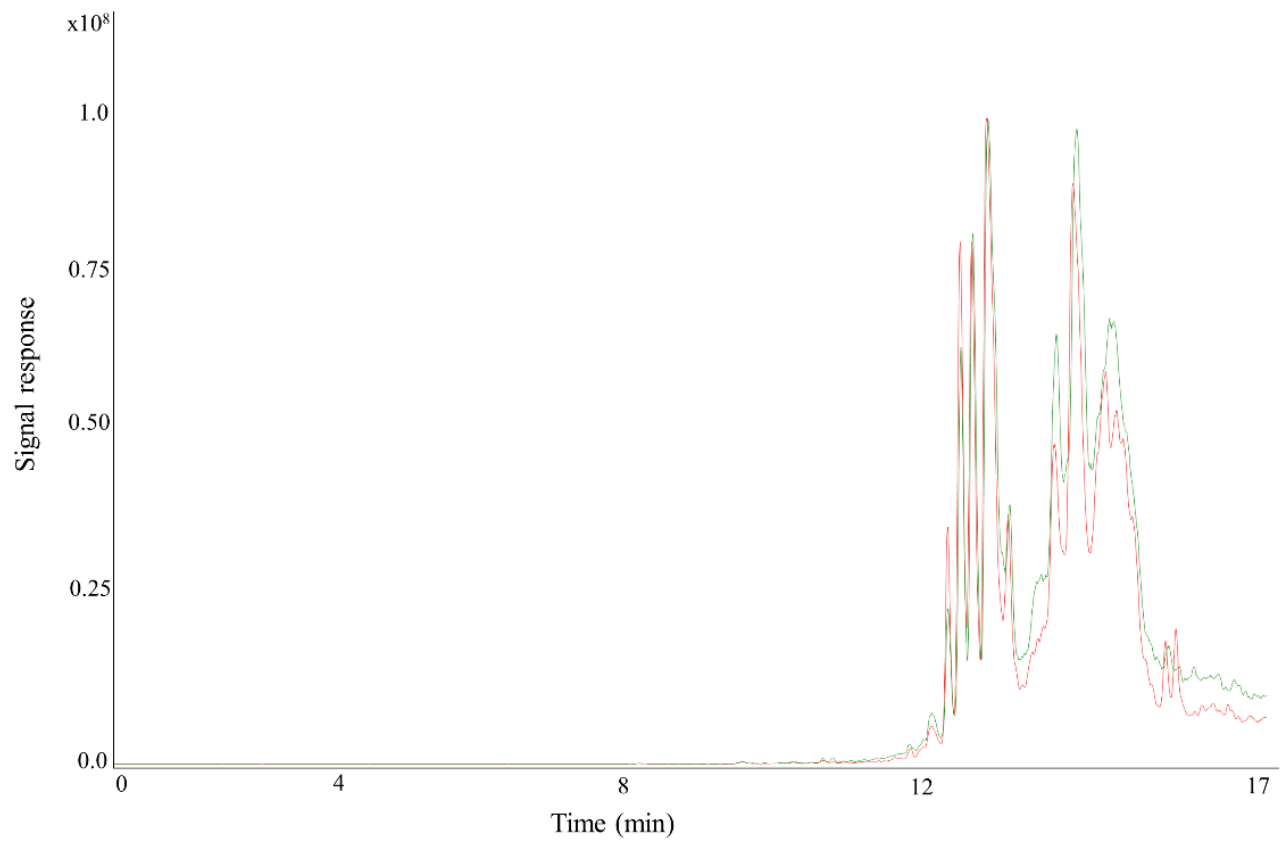

Figure S-1. The overlaid total ion chromatograms of DBS (green color) and plasma (red color) samples obtained from precursor ion scan m/z 184 . 

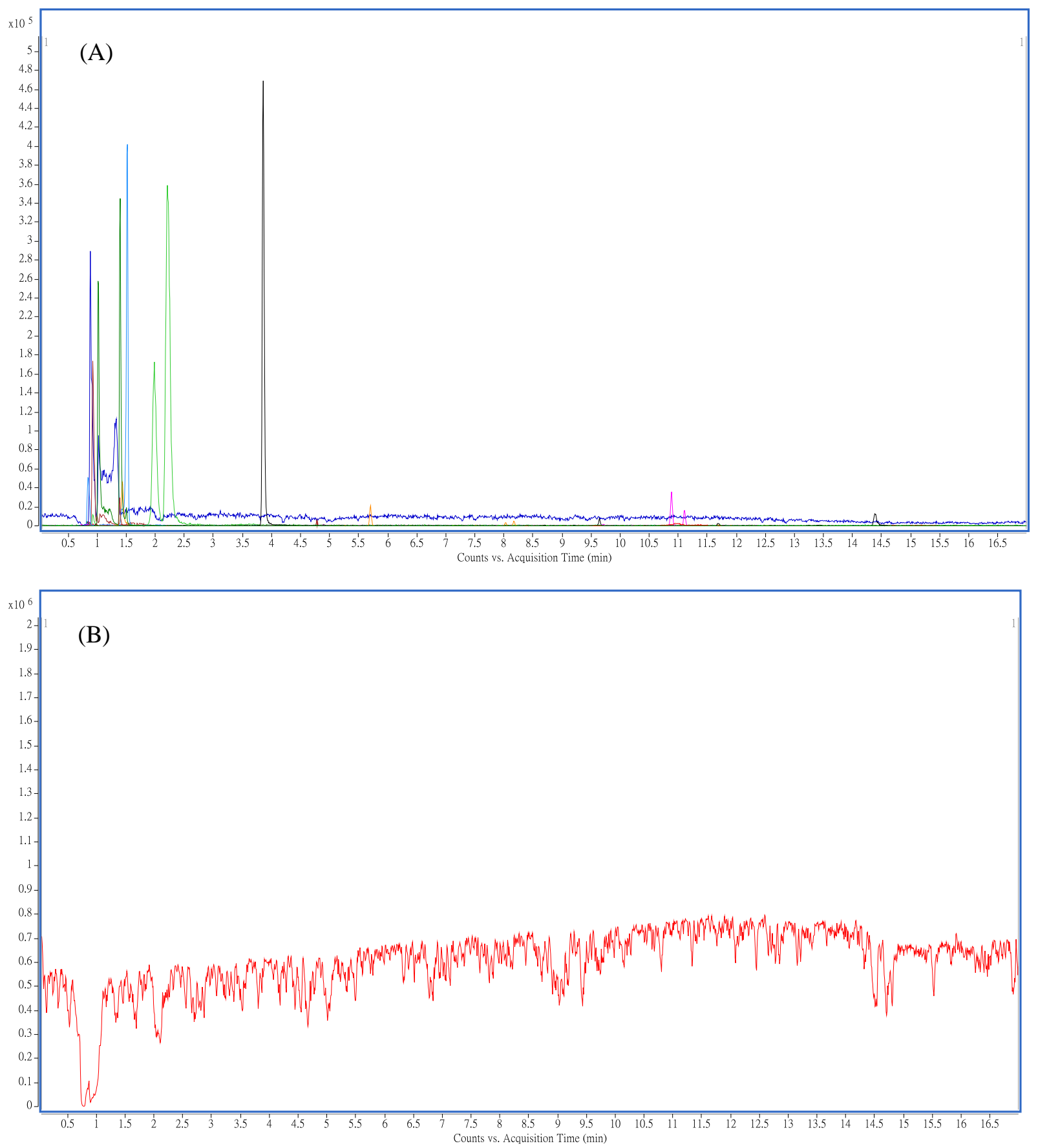

Figure S-2. (A) The representative LC-MS chromatogram of target metabolites and (B) the PCIIS chromatogram for the PCI-IS method. 

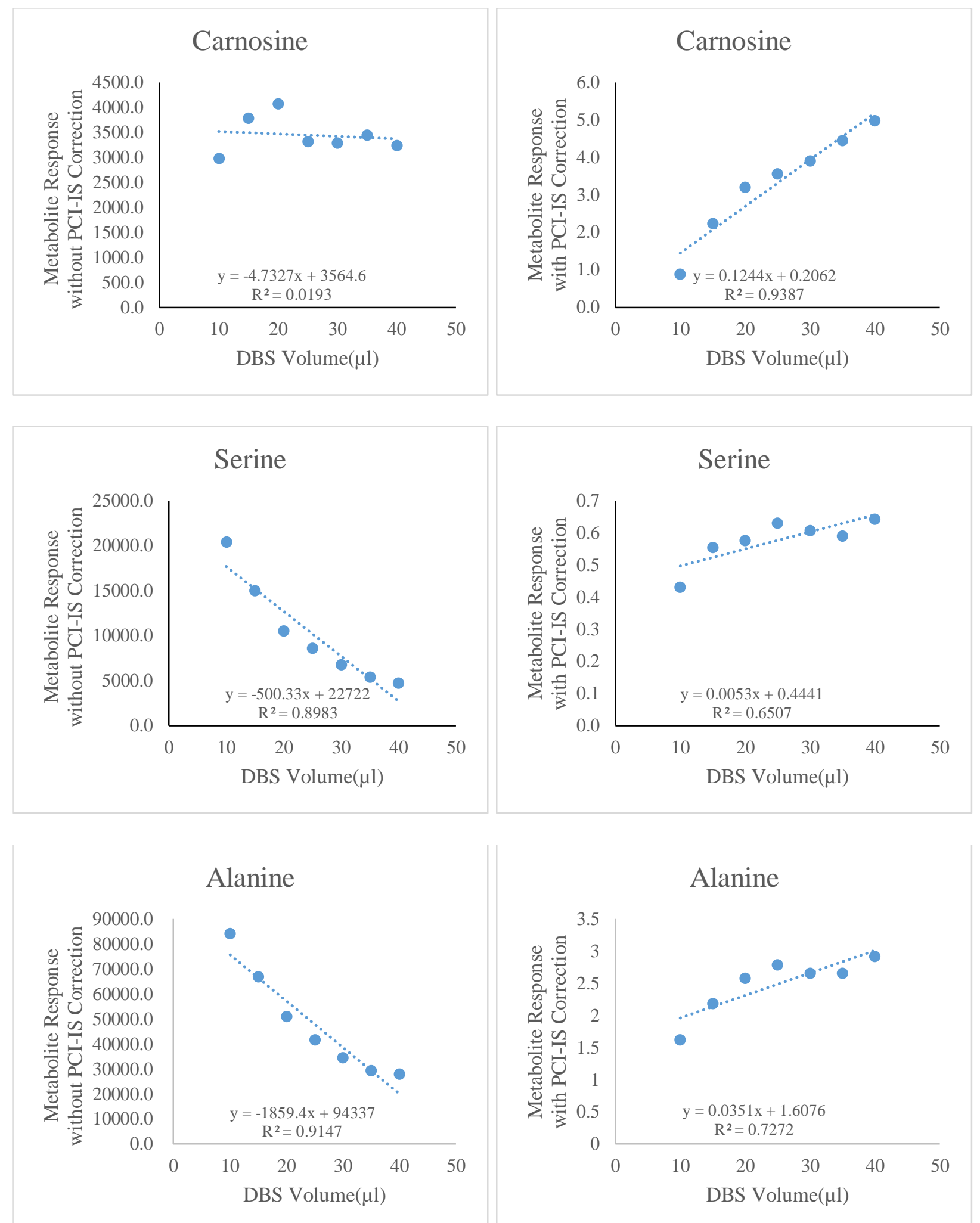

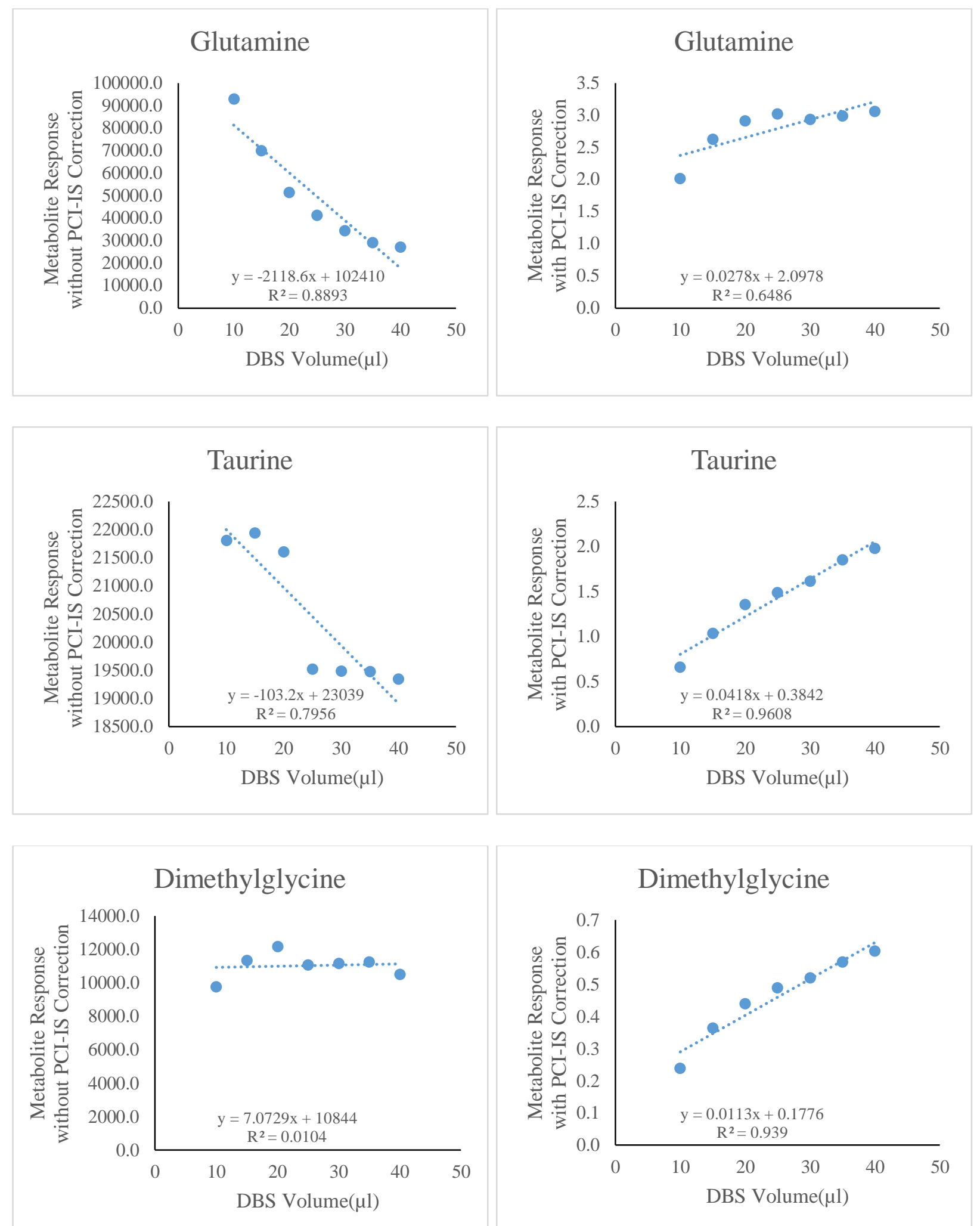

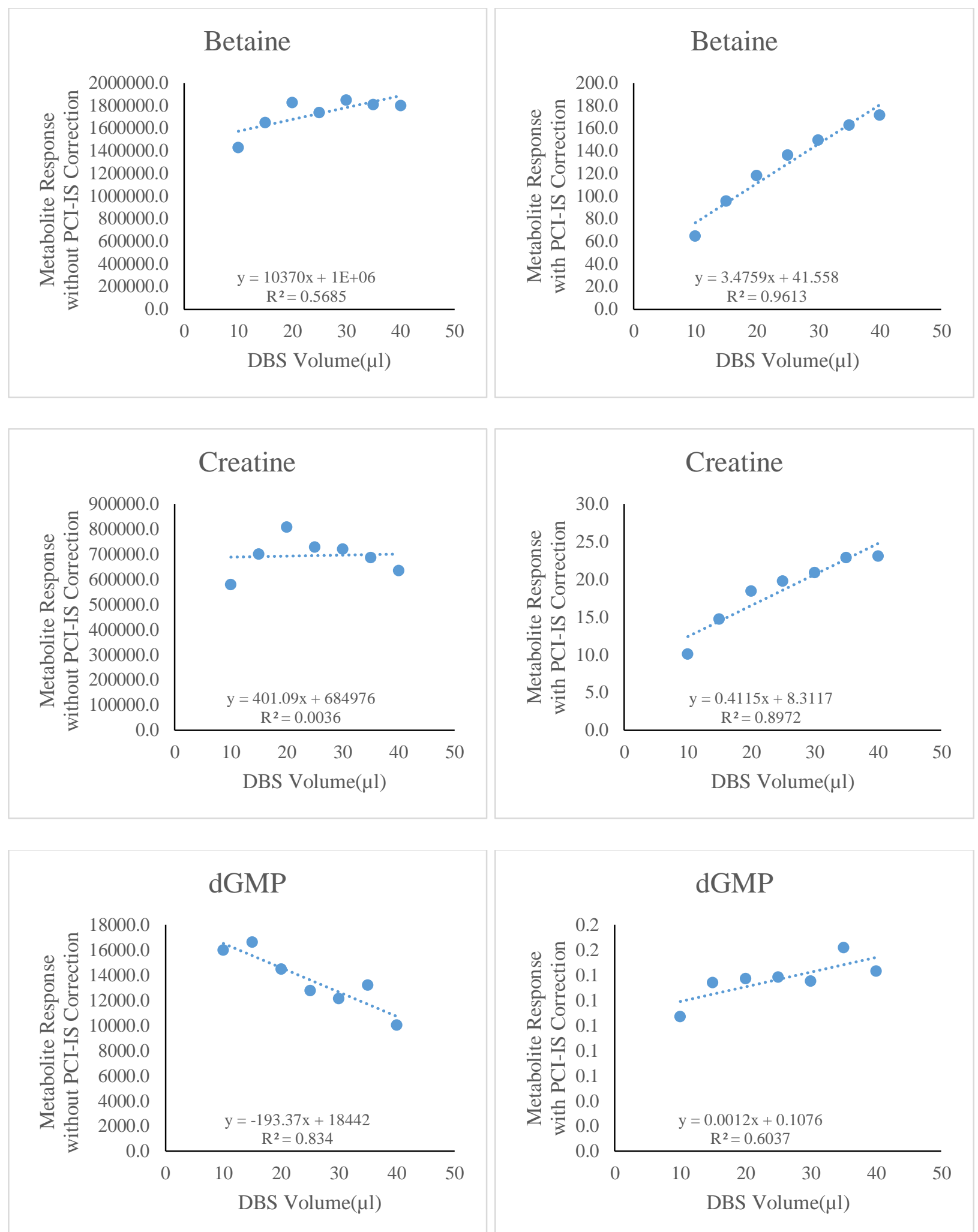

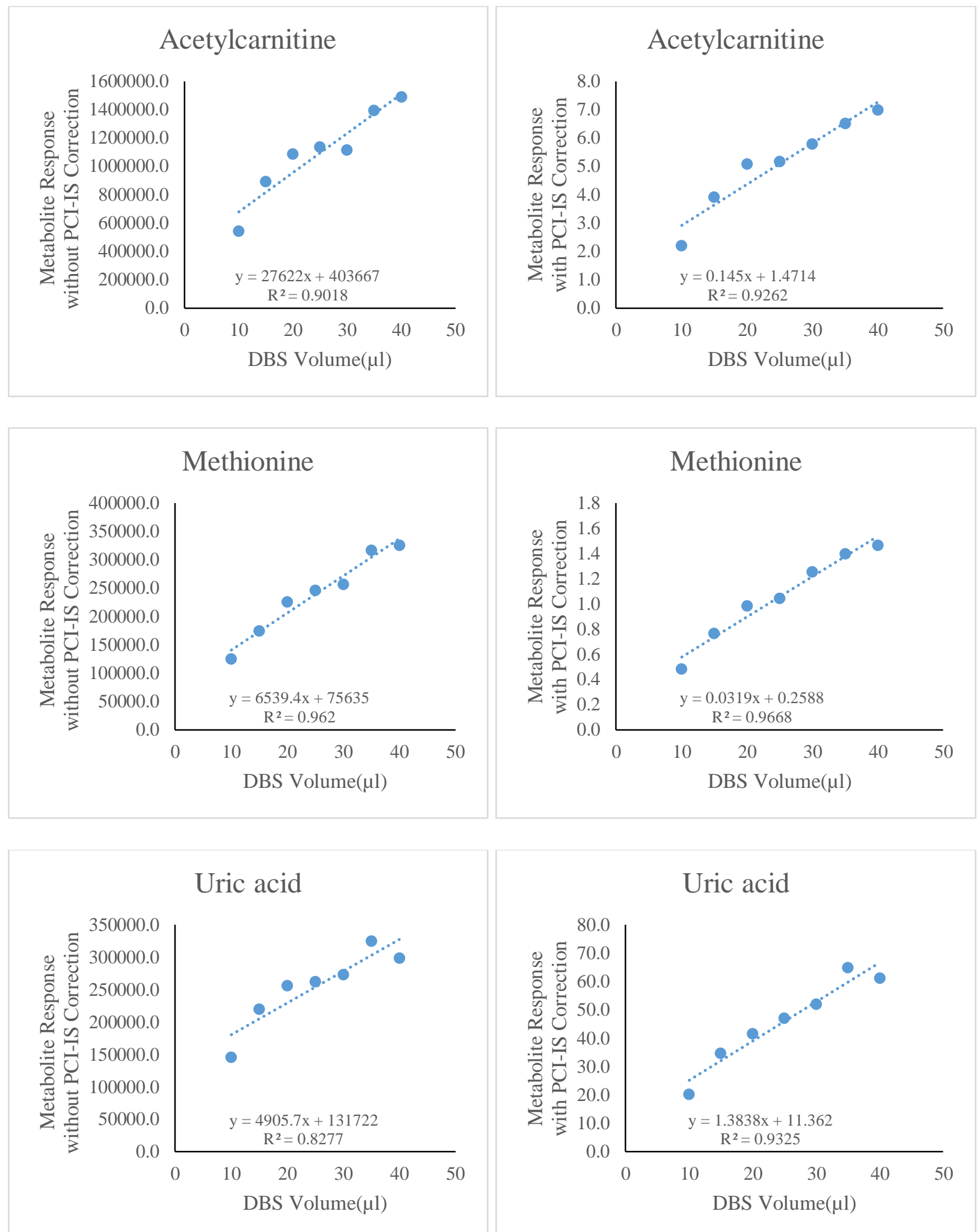

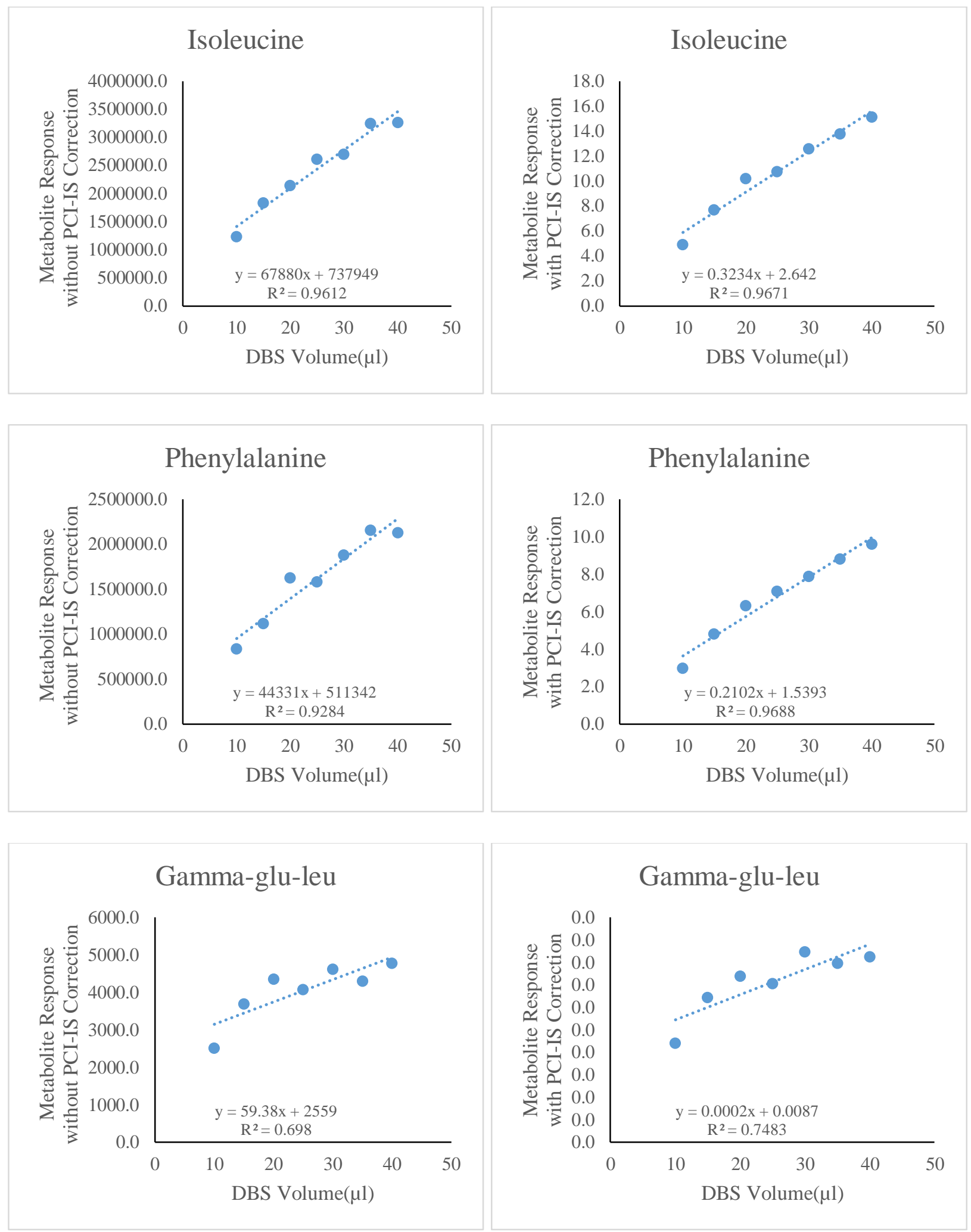

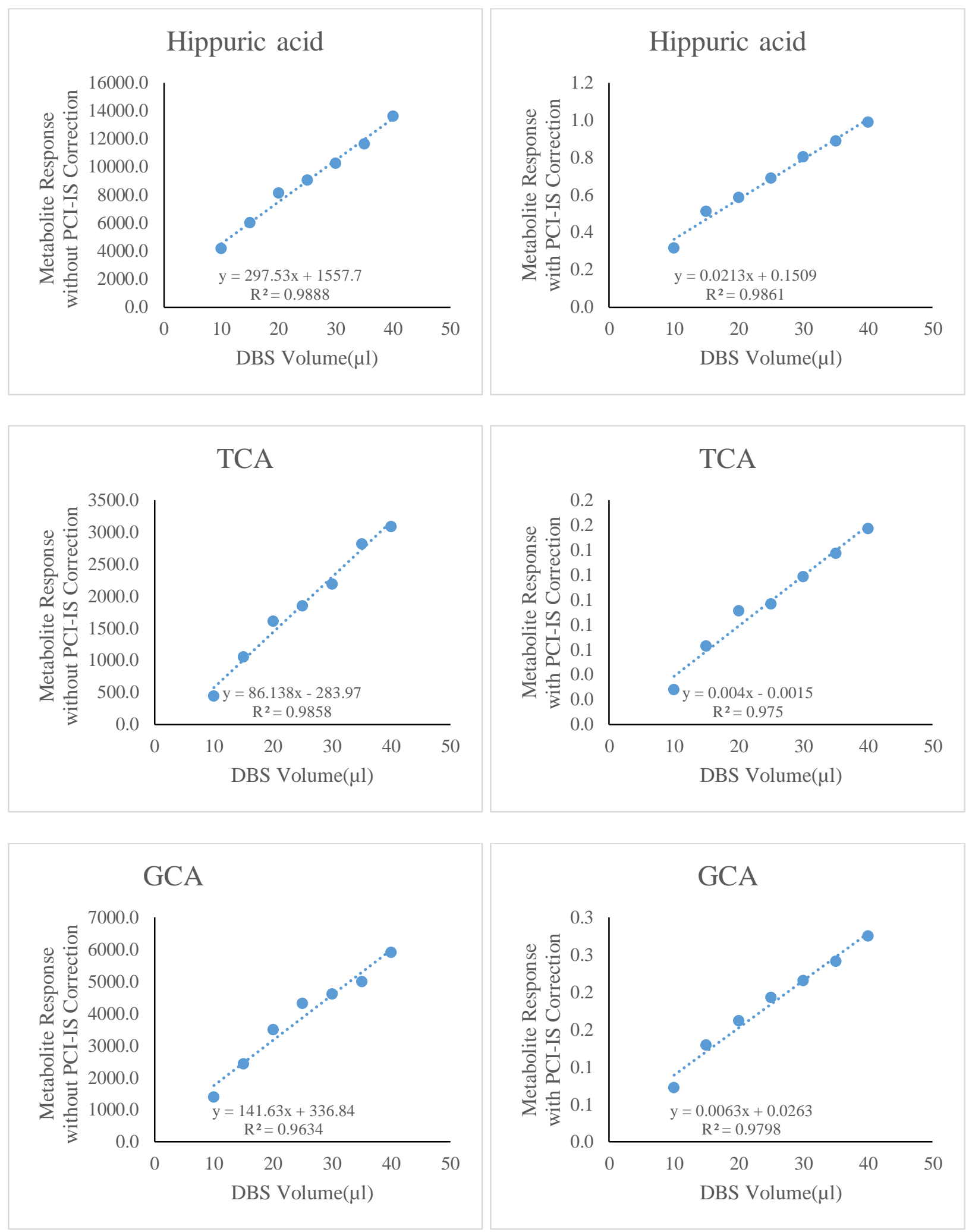

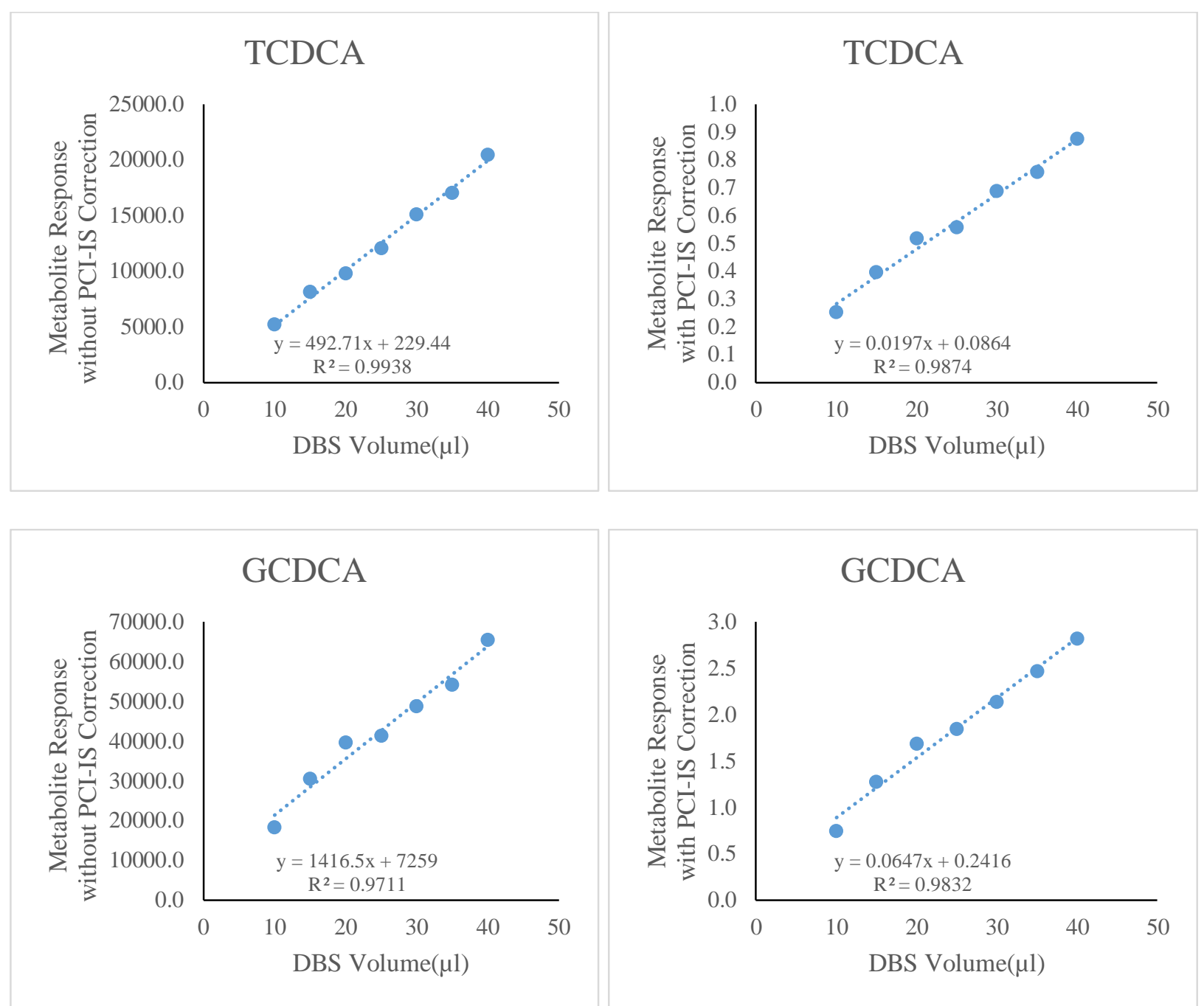

Figure S-3. Correlations between signal intensity and DBS spot volume before and after PCI-IS correction. 
Table S-1. Metabolites with significantly different levels ( $p$-values $<0.05$ ) in DBS samples between APAP-induced liver toxicity and control groups.

\begin{tabular}{lcccc}
\multicolumn{1}{c}{ compound name } & \multicolumn{2}{c}{ with PCI-IS correction } & \multicolumn{2}{c}{ without PCI-IS correction } \\
& fold change & $\boldsymbol{p}$-value & fold change & $\boldsymbol{p}$-value \\
\hline betaine & 1.88 & $1.35 \mathrm{E}-07$ & 1.81 & $2.39 \mathrm{E}-05$ \\
creatinine & 0.61 & $6.47 \mathrm{E}-07$ & 0.64 & $1.49 \mathrm{E}-05$ \\
creatine & 0.73 & $9.37 \mathrm{E}-05$ & 0.70 & $2.02 \mathrm{E}-03$ \\
corticosterone & 2.23 & $1.55 \mathrm{E}-04$ & 2.31 & $5.85 \mathrm{E}-05$ \\
histidine & 1.48 & $2.12 \mathrm{E}-04$ & 0.60 & $4.51 \mathrm{E}-03$ \\
methionine & 1.37 & $2.45 \mathrm{E}-04$ & 1.40 & $2.40 \mathrm{E}-04$ \\
pyruvate & 0.66 & $8.09 \mathrm{E}-03$ & 0.65 & $1.34 \mathrm{E}-04$ \\
ureidopropionic acid & 1.46 & $2.99 \mathrm{E}-04$ & 1.45 & $9.18 \mathrm{E}-03$
\end{tabular}

